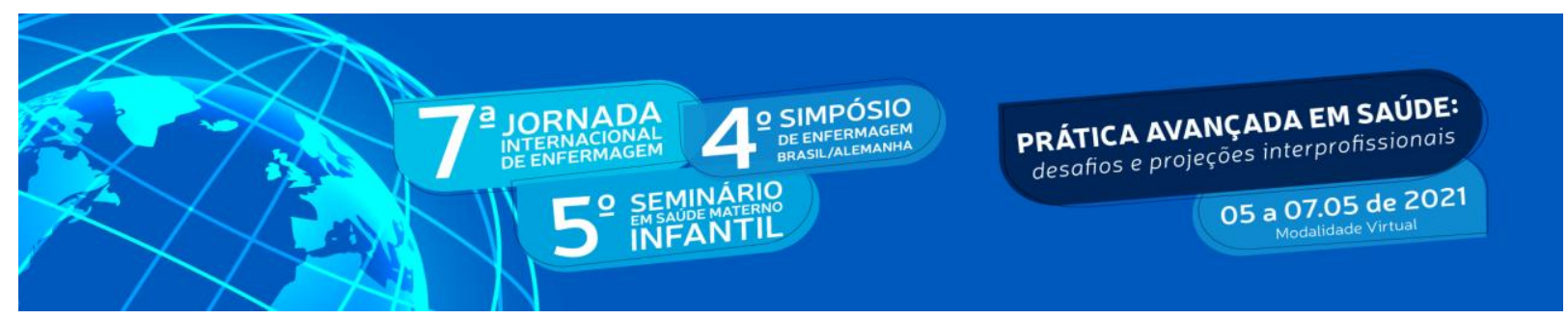

DOI: http://doi.org/10.48195/jie2021-075

\title{
PROCESSO DE ADOECIMENTO POR CÂNCER EM IDOSOS: ABORDAGEM TEÓRICA-REFLEXIVA1
}

\section{Natanna da Rosa²; Eduardo Marques Machado³ Claudia Maria Ferrony Rivas4; Karol Tolfo5; Maria Helena Gehlen6; Naiana Oliveira dos Santos7.}

\begin{abstract}
RESUMO
O atendimento dos idosos com câncer torna-se algo complexo por trazer em si uma doença amedrontadora e uma etapa da vida já estigmatizada em nossa sociedade. Esse trabalho objetiva refletir acerca do processo de adoecimento por câncer em idosos. A metodologia utilizada foi um estudo teórico-reflexivo, sustentado pela literatura científica no Google Scholar, Scielo e Lilacs. A maneira em que o idoso percebe o enfrentamento da doença pode variar de acordo com suas características pessoais, como cultura, hábitos, espiritualidade e valores, assim, influenciando no tratamento e a forma que o mesmo é acolhido. Desse modo, para o cuidado ser realizado de forma integral cabe aos profissionais de saúde voltarem suas ações para às dimensões físicas, psicossociais, emocionais e espirituais, ampliando a forma de cuidar, compreendendo os aspectos em que o paciente idoso está inserido.
\end{abstract}

Palavras-chave: Câncer; Enfermagem; Envelhecimento.

\begin{abstract}
The care of the elderly with cancer becomes something complex because it brings with it a frightening illness and a stage of life already stigmatized in our society. This work aims to reflect on the process of becoming ill with cancer in the elderly. The methodology used was a theoretical-reflective study, supported by the scientific literature on Google Scholar, Scielo and Lilacs. The way in which the elderly perceive coping with the disease can vary according to their personal characteristics, such as culture, habits, spirituality and values, thus influencing the treatment and the way it is received. Thus, for the care to be carried out in an integral way, it is up to the health professionals to turn their actions to the physical, psychosocial, emotional and spiritual dimensions, expanding the way of caring, understanding the aspects in which the elderly patient is inserted.
\end{abstract}

Keywords: Cancer; Nursing; Aging.

\footnotetext{
1 Trabalho de pesquisa do Núcleo de Ensino, Extensão e Pesquisa do Processo de Envelhecimento.

2 Enfermeira especialista em Oncologia. E-mail: natannarosa0509@gmail.com

3 Estudante do Curso de Enfermagem. Universidade Franciscana. E-mail: eduardomarques051@ gmail.com

4 Estudante do Curso de Enfermagem. Universidade Franciscana. E-mail: claudiamfrivas@gmail.com

${ }^{5}$ Estudante do Curso de Enfermagem. Universidade Franciscana. E-mail: karoltolfo98@ gmail.com

6 Orientadora. Doutora. Professora do Curso de Enfermagem. Universidade Franciscana. E-mail: mah@ufn.edu.br

7 Orientadora. Doutora. Professora do Curso de Enfermagem. Universidade Franciscana. E-mail: naiana.santos@ufn.edu.br
} 


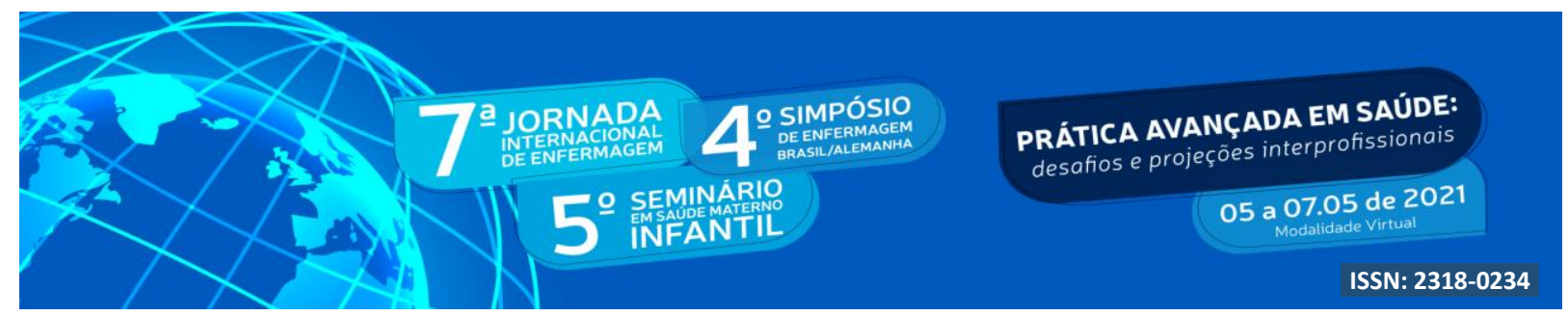

\section{INTRODUÇÃO}

O envelhecimento é um dos principais fatores de risco para que haja o desenvolvimento das doenças crônicas, entre elas, o câncer. As neoplasias representam mais de $45 \%$ dos óbitos em indivíduos acima de 80 anos, com tendência a um aumento gradativo de suas taxas de mortalidade. $\mathrm{O}$ envelhecimento populacional resulta nas incapacidades e em mais doenças crônico-degenerativas, associadas em fatores socioeconômicos e de saúde pública (RIBEIRO, 2018).

Segundo Oliveira (2015), a morbi-mortalidade dessa população vem ocorrendo de forma acelerada no Brasil e acompanha aumento da longevidade dos indivíduos, considerados os idosos longevos de 80 anos ou mais de idade sendo a parcela da população que mais cresce rapidamente.

Ao longo da história, o câncer é concebido como uma doença fatal. Mesmo com os avanços tecnológicos e médicos na área da cirurgia, da farmacologia e da radioterapia direcionados a seu tratamento, essa doença ainda pressupõe a ideia de morte. Assim, o diagnóstico desta enfermidade traz a possibilidade de morte para o paciente. Essa experiência, geralmente, é acompanhada de angústia, desesperança, isolamento e temores que perpassam o desenrolar do tratamento para paciente e seus familiares (ROCHA, 2014).

O adoecimento por câncer pode ser considerado benigno ou maligno, que consiste no crescimento desordenado de células. A doença, na maioria exige tratamentos longos e intensos que são acompanhados da perda da qualidade de vida e desempenho de atividades (BARBOSA; FRANCISCO, 2007). Assim, após essa determinação irá ser delineado a forma em que o tratamento prosseguirá, preservando a qualidade de vida da pessoa idosa.

O câncer por si só ser uma doença que "amedronta", juntamente com a velhice que já é vista como uma etapa da vida que é estigmatizada, nesse sentido, o atendimento aos idosos portadores de câncer torna-se complexo (ROCHA, 2014). Assim, os profissionais de enfermagem possuem papel relevante no tratamento oncológico desse paciente, seguindo o acompanhamento psicológico, físico e social durante todo o processo.

Nesse sentido, é de suma importância que o paciente tenha conhecimento do seu estado de saúde, bem como, é realizado o tratamento a fim de que se tenha condições de como irá conduzir o processo. Ademais, cabe-se compreender a autonomia do paciente idoso com 


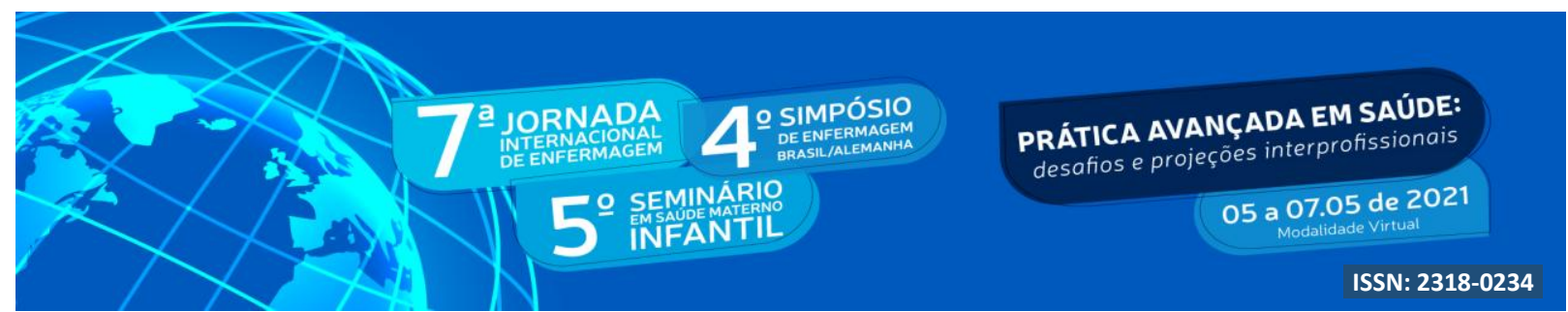

câncer faz-se complexa, uma vez que o processo do adoecimento impõe limitações que por vezes dificultam o exercício desse direito (VISENTIN, 2007).

\section{OBJETIVO}

Refletir acerca do processo de adoecimento por câncer em idosos.

\section{METODOLOGIA}

Trata-se de um estudo teórico-reflexivo, sustentado pela literatura científica. Utilizando-se dados bibliográficos baseados na Literatura Latino-Americana e do Caribe em Ciências da Saúde (LILACS), Google Scholar e Scielo, nos meses de fevereiro e março de 2021. Foram utilizados como palavras chaves: envelhecimento; câncer; percepções; enfermagem.

Quanto aos critérios de inclusão considerou-se: os artigos completos disponíveis em Português que abordassem a temática com recorte temporal de 5 (cinco) anos. Os critérios de exclusão foram artigos que não estivessem disponíveis na íntegra, teses, dissertações e os que não atendessem o objetivo proposto no trabalho. A questão da pesquisa na literatura abordou o que se tem produzido acerca do processo de adoecimento por câncer em idosos.

\section{RESULTADOS E DISCUSSÃO}

A análise e interpretação dos dados levantados foram realizadas na forma descritiva, de forma a reunir os resultados principais dos estudos incluídos em relação à questão de pesquisa.

Nesse sentido foram selecionados 7 (sete) artigos para embasar a reflexão das percepções dos idosos frente ao adoecimento por câncer.

Observou-se através da literatura selecionada que com o aumento da expectativa de vida da população brasileira se tem o aumento das taxas de pacientes idosos com câncer. Nessa perspectiva, às doenças crônico-degenerativas vem substituindo às infectocontagiosas nas taxas e no perfil de morbimortalidade do país (ANDRADE, 2018). 


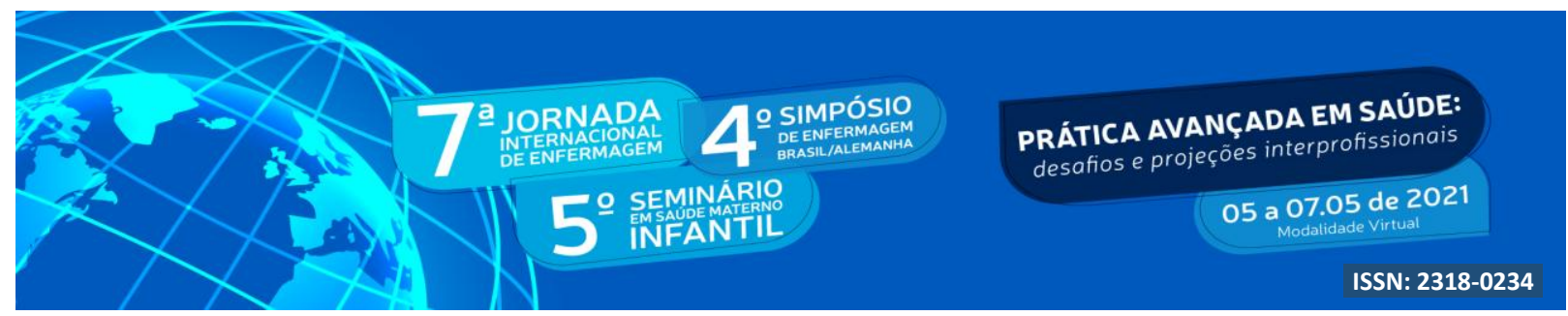

Por vezes o diagnóstico de câncer, no imaginário de paciente e família, está atrelado a estereótipos e mitos no que se refere ao tratamento e prognóstico. Frequentemente o câncer é também associado a morte, a dor e a procedimentos invasivos e mutiladores o que pode levar ao sofrimento psíquico os que enfrentam a doença (DI MENEZES; LIMA, 2019).

Pesquisa realizada sobre a percepção do câncer por idosos, pondera que idosos com idade menor ou igual a 80 anos tem maior possibilidade de crer que os exames feitos de modo regular podem identificar a doença em seu estágio inicial. Já idosos com histórico positivo da doença, demonstraram maior chance de acreditar que poucos sobrevivem ao câncer. A pesquisa também constatou que todos os idosos entrevistados com diagnóstico prévio de câncer reconheceram que a doença pode ser curada, desde que detectada cedo (BRAZ et al, 2018).

O diagnóstico de câncer para a pessoa idosa pode vir acompanhado de mudanças na vida e limitações com sentimentos de perda, ansiedade e depressão. Essas mudanças acabam diminuindo a qualidade de vida e limitações em suas atividades, podendo variar significativamente de acordo com a cultura. Assim, a enfermagem se torna responsável pela criação de um plano de cuidados, dando autonomia ao idoso e estimulando atividades que proporcionem recuperação da qualidade de vida, no sentido biopsicossocial (SILVA, HANSEL, DA SILVA, 2016).

A maneira em que o idoso percebe o enfrentamento do tratamento pode variar de acordo com suas características pessoais, como cultura, hábitos e valores. Assim, influenciando no tratamento e a forma que o mesmo é acolhido e amparado no enfrentamento da doença (MELO; BARBOSA, 2015).

É importante que o profissional da saúde para efetivar o cuidado ao idoso com câncer saiba escutar e olhar, compreendendo o paciente e sua família, as queixas e indagações em relação ao contexto vivenciado, auxiliando a lidar e enfrentar a doença e o processo do tratamento (GRIPA et al, 2018).

A partir do diagnóstico de câncer, os pacientes têm muitos desafios a serem enfrentados, se tratando de idoso se tem o processo de envelhecimento como um fator de vulnerabilidade. Assim, cabe o cuidado de forma ampliada, acolhendo esse paciente que culturalmente já tem medo em realizar o tratamento por mitos culturais. 


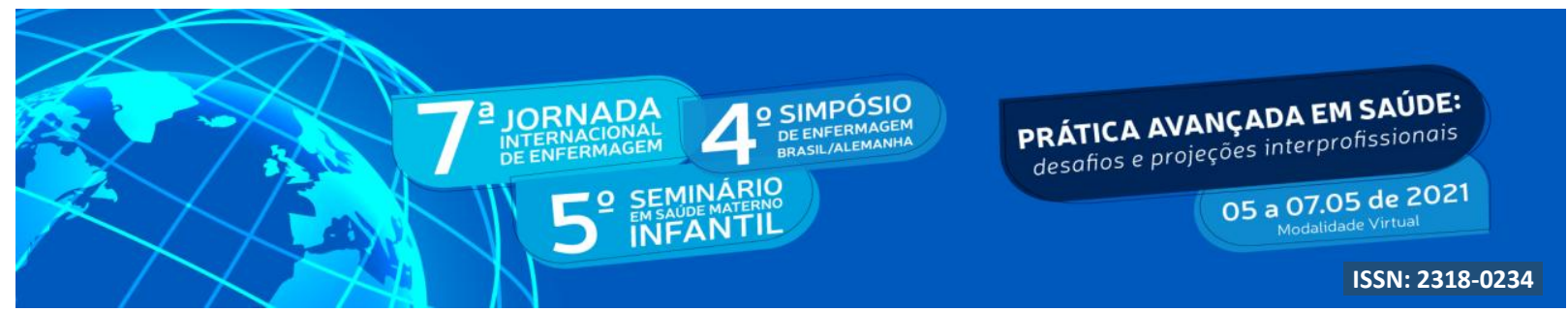

Os idosos com neoplasias tem uma diminuição da qualidade de vida e aumento da ansiedade. Com isso, cabe os profissionais inseridos nos serviços que atendem essa população ter conhecimento acerca dos aspectos sociais, físicos e emocionais. Assim, realizar um planejamento de cuidado, proporcionando qualidade de vida e atendendo às subjetividades do idoso (DOS SANTOS; SILVA; SALDANHA, 2020).

A diminuição da qualidade de vida devido a neoplasia junto ao envelhecimento é um fator que necessita de atenção dos serviços de saúde. Visto que o cuidado não deve estar voltado apenas ao câncer, mas prestar apoio emocional e social. Promovendo ferramentas para que o sujeito enfrente o tratamento.

Assim, a capacitação dos profissionais torna-se imprescindível para práticas direcionadas ao cuidado de pacientes idosos, buscando assegurar o direito do paciente a um atendimento e tratamento qualificado que fomente a autonomia sobre as decisões a ser tomadas em relação a doença e ao tratamento (DI MENEZES; LIMA, 2019).

No cuidado são utilizados diversos componentes, entre esses a espiritualidade. Os idosos diversas vezes tem o lado espiritual muito forte no enfrentamento de uma doença. Assim, a colocação da espiritualidade no amparo e fortalecimento, reduzindo a ansiedade, aflição, dos pacientes oncológicos. Sendo a espiritualidade subjetiva e cabendo aos profissionais respeitar de forma humana às diversas forma de enfrentamento (ALVES; PAULA, 2016).

No que se refere a qualidade de vida ao paciente idoso em tratamento oncológico e/ou cuidados paliativos, para proporcionar conforto aos pacientes a equipe deve realizar a escuta qualificada (ALVES; PAULA, 2016). Com isso, envolvendo o bem estar físico, psicológico, emocional e espiritual, proporcionando atenção multidimensional.

\section{CONCLUSÃO}

Diante do exposto, o envelhecimento vem carregado de morbi-mortalidade, com o aumento das doenças crônico-degenerativas, resultando em incapacidades temporárias ou permanentes. Assim, no processo de cuidado ao paciente oncológico, se tem com o objetivo de promover a manutenção do bem estar e qualidade de vida. 


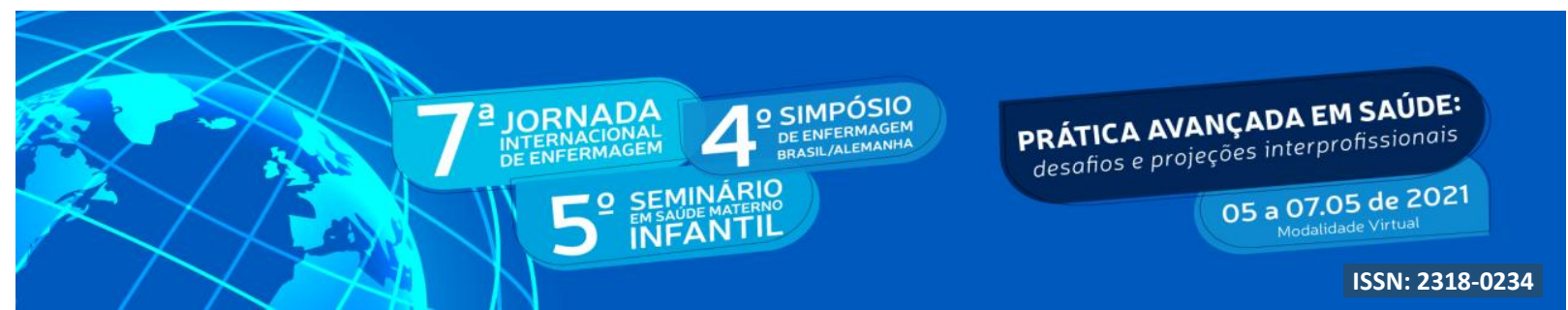

Para que o cuidado ser realizado de forma integral cabe aos profissionais de saúde voltarem suas ações para às dimensões físicas, psicossociais, emocionais e espirituais.

Diante da complexidade da temática, conclui-se que ainda há que se avançar no processo de estruturação de um cuidado integral e humanizado no cuidado de idosos no processo de adoecimento por câncer. Desta forma, se faz necessário a presença de profissionais qualificados para atender essas demandas, promovendo uma rede de apoio ao paciente e a sua família.

\section{REFERÊNCIAS}

ALVES, J. P. S.; PAULA, M. F. C. A. Espiritualidade na Arte do Cuidar: experiência do idoso hospitalizado com câncer. Atas - Investigação Qualitativa em Saúde, vol. 2, Portugal, 2016.

ANDRADE, L. (Org.). Territórios de exclusão: população idosa e cuidados paliativos na cidade de São Paulo. Editora Alumiar, São Paulo, 2018.

BARBOSA, L. N. F.; FRANCISCO, A. L. A subjetividade do câncer na cultura: implicações na clínica contemporânea. Revista da SBPH, v. 10, n. 1, p. 9-24, São Paulo, 2007.

BRAZ, I. F. L. et al. Análise da percepção do câncer por idosos. Einstein, v. 16 n. 2, 2018.

DI MENEZES, N. R. C.; LIMA, P. M. R. Envelhecimento e doença crônica: uma análise da autonomia decisória de pacientes idosos com Mieloma Múltiplo. Rev. SBPH, v. 22, n. 1, p. 107-126, São Paulo, 2019.

DOS SANTOS, A. L. S.; SILVA, L. M.; SALDANHA, Z. O. Idosos com câncer no período pré-operatório: dados de qualidade de vida, ansiedade e depressão. Rev Pan Amaz Saude, vol. 11:e202000490, Ananindeua, 2020.

GRIPA, J. A. et al. Cuidado humanizado de enfermagem à pessoa idosa com câncer. Disciplinarum Scientia. Série: Ciências da Saúde, Santa Maria, v. 19, n. 2, p. 235-243, 2018.

MELO, D. M.; BARBOSA , A. J. G. O uso do MiniExame do Estado Mental em pesquisas com idosos no Brasil: Uma Revisão Sistemática. Ciência, \& Saúde Coletiva, v.20, p.38653876, 2015.

OLIVEIRA, T. C.; MEDEIROS, W. R.; LIMA, K. C.. Diferenciais de mortalidade por causas nas faixas etárias limítrofes de idosos. Rev. bras. geriatr. gerontol., Rio de Janeiro, v. 18, n. 1, p. 85-94, Mar. 2015. 


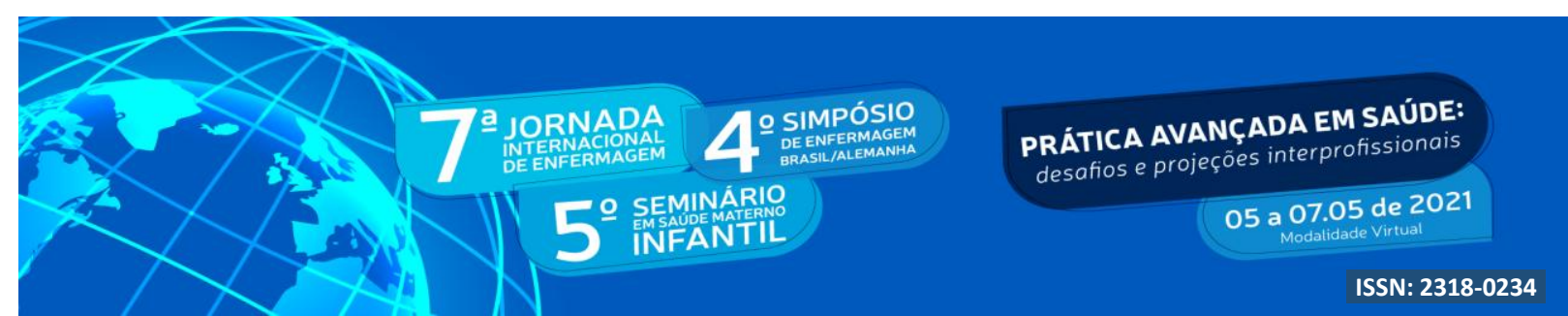

RIBEIRO, J. R.; POLES, K. Cuidados Paliativos: Prática dos Médicos da Estratégia Saúde da Família. Rev. bras. educ. med., Brasília, v. 43, n. 3, p. 62-72, July 2019.

RIBEIRO, M. S.; BORGES, M. S. Percepções sobre envelhecer e adoecer: um estudo com idosos em cuidados paliativos. Rev. bras. geriatr. gerontol., Rio de Janeiro, v. 21, n. 6, p. 701-710, Dec. 2018.

ROCHA, L. S. et al . O cuidado de si de idosos que convivem com câncer em tratamento ambulatorial. Texto contexto - enferm., Florianópolis , v. 23, n. 1, p. 29-37, Mar. 2014.

SILVA, J. A.; HANSEL, C. G.; DA SILVA, J. Qualidade de vida na perspectiva de idosos com câncer: implicações para enfermagem na atenção básica. Rev enferm UERJ, vol. 24, n. 3:e9621, Rio de Janeiro, 2016.

VISENTIN, A.; LABRONICI, L.; LENARDT, M.H. Autonomia do paciente idoso com câncer: o direito de saber o diagnóstico. Acta Paulista de Enfermagem, São Paulo, v. 20, n. 4, p. 509-13, 2007. 